\title{
Implications of Bovine beta casein subtypes in health and homeostasis
}

\author{
M.S. Trivedi ${ }^{1}$ and A.J. Clarke ${ }^{2}$ \\ ${ }^{1}$ Northeastern University, Boston, USA and ${ }^{2}$ A2 Corporation, Auckland, New Zealand
}

Milk is a whole-food with numerous nutritive benefits. And in some cases i.e. during early developmental stages, milk and/or milkbased formulas are important and only source of nutrition. In spite of this critical significance, casein free diet is recommended for infants with immunological sensitivities, gastrointestinal (GI) issues or neurodevelopmental disorder ${ }^{(1,2)}$. However, rationale for such dietary intervention is still unelucidated, preventing sensitive infants from all-round nutritive benefits of milk.

$\beta$-casein (about 25-30\% of cows' milk proteins) occurs in several different forms determined based on their inherent DNA-sequence; i.e. A1, A2, A3, B, C etc, yet original form of bovine $\beta$-casein is A2, which is more similar to all other known $\beta$-casein in mammals. A1 and A2 have one amino acid difference in it's structural sequence, which affects the resultant secondary structure as well as it's enzymatic cleavage, i.e. A1 but not A2 $\beta$-casein liberates a heptapeptide $\beta$-casomorphin-7 (BCM7; YPFPGPL). Epidemiological studies indicate elevated levels of BCM-7 found in blood and urine samples of patients with neurological defects ${ }^{(3)}$. Recent evidence also indicates a direct correlation between GI issues and consumption of A1 milk ${ }^{(4)}$ and not A2 milk.

The current research uses neuronal and intestinal cell lines to indicate that BCM-7 can alter metabolic homeostasis measured using HPLC. Changes in metabolic flux further translate into altered gene regulation i.e. epigenetic changes measured across the entire genome. This was statistically significant at transcription start site, which controls gene transcription. This resulted in altered molecular machinery and gene expression levels; especially for genes implicated in GI function. Thus, altered metabolic flux can translate into differential developmental trajectory or health conditions, including immunological response and GI function. However, such changes or associations were not observed in our current human studies or animal studies with A2 $\beta$-casein for any of these diseases.

Table 1. SH-SY5Y human neuroblastoma cells were treated with $1 \mu \mathrm{M}$ morphine, bBCM7 for 4 hours $(\mathrm{n}=5)$. DNA methylation was measured by MBD-seq, mRNA transcription was measured with whole genome microarray. IPA functional analysis performed with DETs and DMTs for the treatments vs. control. P values were generated by IPA for gene ontology categories associated with gastrointestinal disease, inflammatory disease or inflammatory response for each treatment vs. control. P-values are displayed as a range based on the number of genes from the experimental list that appears in a particular ontology within Disease category

\begin{tabular}{|c|c|c|c|c|}
\hline \multirow[b]{2}{*}{ Disease or Disorder } & \multicolumn{2}{|c|}{ P-value Ranges for DMTs } & \multicolumn{2}{|c|}{ P-value Ranges for DETs } \\
\hline & Morphine & bBCM7 & Morphine & bBM7 \\
\hline Gastrointestinal Disease & 9.34E-08-1.07E-02 & & & $4 \cdot 06 \mathrm{E}-08-1 \cdot 33 \mathrm{E}-03$ \\
\hline $\begin{array}{l}\text { Inflammatory Disease } \\
\text { Inflammatory Response }\end{array}$ & $5.46 \mathrm{E}-05-1.07 \mathrm{E}-02$ & $3.96 \mathrm{E}-03-4.51 \mathrm{E}-02$ & 1.33E-12-1.35E-03 & \\
\hline
\end{tabular}

Hence, current study elucidates the health implications of different bovine beta-caseins. However, the current research is still preliminary and it is noteworthy to mention that concrete evidence is still sought for causal link between A1 consumption and other diseases as well as illuminating other benefits of A2 beta casein milk.

1. Millward C, Ferriter M, Calver S, Connell-Jones G.. 2004; (2):CD003498.

2. Ashwood P, Anthony A, Torrente F, Wakefield AJ. S. J Clin Immunol. 2004 Nov; 24(6):664-73.

3. Reichelt KL, Knivsberg AM. Nutr Neurosci. 2003 Feb; 6(1):19-28.

4. Ho S, Woodford K, Kukuljan S, Pal S. Eur J Clin Nutr. 2014 Sep; 68(9):994-1000. 